\title{
La folie des uns fait le travail des autres, la vacma et le tramway
}

Robin FOOT

Résumé: L'analyse du dispositif d'homme-mort implanté sur les tramways nous amène à explorer l'imaginaire des concepteurs, exploitants et constructeurs au travers des métamorphoses de cet objet technique. Celui-ci se voit attribué des fonctions fictives et fantasmatiques alors que s'oublie sa fonction originelle de détection de la défaillance. Cette dérive d'un objet technique prend appui, paradoxalement, sur une enquête menée par des médecins en 1965 associés à la CGT SNCF. Cinquante plus tard, une nouvelle lutte, toujours avec la CGT mais dans le transport urbain, vient réinterroger cet homme-mort.

F. Sigaut pose la question de l'«aliénation culturelle », en lien avec la technique, comme situation d'un groupe social qui se coupe du réel qu'il est censé mettre en forme (Sigaut, 1990). Les conséquences de cette aliénation culturelle dans le monde du travail ont été développées par C. Dejours pour rendre compte de ces situations où «le réel reste forclos, cependant que la direction et les cadres débattent de questions de gestion, de management et de doctrine tout en étant coupés du réel du travail » (Dejours, 1993, p. 249). B. Latour parle également de cette " "altération du rapport au monde" (...) terme savant pour designer la folie » (Latour, 2015, p. 19) comme d'un problème central de notre monde technique. Cette convergence signale probablement l'importance de cette question de la mise en forme du réel au sein de nos sociétés industrielles dont la « folie » n'est qu'une de ses formes limites. 
La plupart des cas mis en discussion montre que le refoulement du réel prend sa source dans des divergences d'intérêt où le retour du réel peut mettre en danger la position d'un acteur dominant (Amalberti, 1996; Llory, 1996; Henry, 2007; de Terssac \& Mignard, 2011; Proctor, 2014). Nous voudrions, dans cet article, sans nier la réalité de ces situations, explorer un cas, où aucun intérêt économique, aucun espoir de bénéfice, a priori, ne pousse les responsables à configurer un dispositif, le système d'homme-mort des tramways, de telle sorte qu'il impose des contraintes élevées aux conducteurs et mette en cause leur santé et la sécurité de la conduite.

Cette situation, désengagée d'un conflit d'intérêt, constitue une sorte de cas d'école pour comprendre la manière dont un objet technique peut perdre son rapport au réel de l'activité et se constituer, dans un milieu technico-industriel, autour d'un fantasme. Parler de milieu signifie que l'on a à faire non pas à une organisation hiérarchique unidimensionnelle mais à un espace social instancié par un réseau d'acteurs relevant de régime différencié de fonctionnement. Cela va d'organismes d'État à des entreprises exploitantes, des constructeurs et des autorités organisatrices. La liste n'est pas exhaustive.

Pour passer d'un réseau d'acteurs hétérogènes à un acteur-réseau (Law, 1992), il est nécessaire que les différents acteurs qui le composent s'accordent sur la définition des objets qu'ils vont mobiliser dans l'action. On peut dès lors suivre le lent processus de mise en forme d'un objet technique par les mots employés et les théories qui lui sont associées. On se rend compte alors que les mots ne réfèrent pas à un réel qui serait déjà-là mais au contraire trahissent «le » réel pour donner à voir et à entendre socialement « un » réel. Magritte parlait de la trahison des images et disait également qu' "un objet ne tient pas tellement à son nom qu'on ne puisse lui en trouver un autre qui lui convienne mieux » (Magritte, 1994, p. 34), la sociologie des sciences parle également de cette fatalité du malentendu dans la traduction du réel dans le langage (Latour, 1984, p. 73).

Ce travail de trahison/traduction du réel se fait toujours au risque que la trahison du réel dans les mots devienne trop forte, contre-productive et qu'elle aboutisse à ne plus pouvoir agir efficacement sur ce réel, que la « performance » du discours dans l'action vienne buter sur l'épreuve du réel. C'est à cette impasse qu'il nous semble que l'on soit parvenu avec l'homme-mort sur les tramways dont la définition fonctionnelle contraint les conducteurs à ne pas maintenir la veille plus de dix secondes tant les responsables craignent que le conducteur soit saisi d'une «défaillance 
crispée ». Or, un tel type de défaillance n'existe que dans l'imagination des responsables car, en termes physiologiques, une défaillance se traduit par « une mollesse de la musculature » (Paillard, 1976).

La folie dont nous parlons à propos de cet homme-mort est à considérer dans la manière dont s'est tissé autour de cet objet un discours qui, pour l'essentiel, semble ne plus avoir à justifier d'un lien avec le réel qui constitue pourtant, en théorie, son plan d'épreuve, plan d'épreuve d'autant plus exigeant qu'il s'agit d'un équipement de sécurité du monde ferroviaire. Le marqueur de la «folie» se situe dans la capacité de cet objet et du groupe social qui le porte, les responsables du tramway et de la sécurité, de se couper d'autres groupes sociaux qui ont aussi leur mot à dire à son propos, les conducteurs, les services de santé et les chercheurs, pour ne citer qu'eux.

Dans cette enquête, nous suivrons la manière dont ce dispositif s'est redéfini au cours de son implantation dans les tramways urbains, comment, au travers de ses successives traductions techniques et sémantiques, de 1985 jusqu'à nos jours, il a perdu le sens des réalités.

\section{Une enquête au long cours sur la VACMA et les tramways}

En 2003, avec Ghislaine Doniol-Shaw, ergonome au LATTS, lors d'une demande d'expertise pour le comité d'entreprise du réseau de ClermontFerrand, nous avons découvert le système d'homme-mort qui devait être implanté sur ce tramway. Il s'agissait d'une vacma (veille automatique à contrôle du maintien d'appui) avec une temporisation très brève -12 secondes maximum de maintien et 2,5 secondes maximum de relâchement - qui ne doit pas être dépassée sinon une alarme sonore retentit pendant 2,5 secondes puis, sans réaction du conducteur, le freinage d'urgence se déclenche. L'étonnement a été notre première réaction.

Car, si un dispositif de veille se justifie pour prévenir un accident dû à l'assoupissement, un malaise ou la mort d'un conducteur, rien ne justifie, une temporisation aussi courte :

"Sur tous les systèmes de veille, ce temps de relâchement autorisé est très court. Et pour moi, des temps de maintien de 10, 15, 30, 50 secondes ou même plus, ça ne veut pas dire grand-chose puisque c'est le relâché qui va signaler la défaillance du conducteur. S'il a un malaise, le système va se déclencher dans le délai, toujours court, 
laissé à la position relâchée » (Mollard in Doniol-Shaw \& Foot, 2004, p. 57).

L'obligation périodique de relâchement simule une défaillance et n'a pour seul enjeu, en théorie, que de prévenir le blocage volontaire du dispositif de veille par les conducteurs. Rien ne justifie donc que l'on oblige les conducteurs à manifester, au moins toutes les dix secondes, qu'ils n'ont pas fraudé le système.

En 2004, à Clermont-Ferrand, il y a un consensus entre les syndicats, la direction et l'équipe d'expertise sur le fait qu'il n'y a aucune justification physiologique à un tel dispositif. Si localement, il y a un accord pour remettre en cause ce dispositif, encore faut-il que cette position soit partagée par le milieu. La décision est prise, conjointement avec la direction du réseau, d'ouvrir un débat public, ouvert à tous les acteurs du tramway, sur le "travail de conduite et la sécurité des tramways" durant lequel la question de la vacma sera posée (Doniol-Shaw \& Foot, 2004).

Lors de ce séminaire, le danger que cette vacma, avec cette temporisation aussi brève, vise à conjurer, a pu être identifié. Le responsable des études fonctionnelles pour le matériel roulant de la RATP l'a parfaitement résumé, même si cela reste en creux de son énoncé. Il s'agit de conjurer le danger d'un malaise qui ne se traduirait pas par un relâchement du « cerclo » ${ }^{1}$, c'est-à-dire d'un « malaise-crispé » :

«On s'est également demandé ce qui pourrait se passer si l'arrêt automatique ne fonctionnait pas, s'il était hors service par exemple, et que le conducteur avait un malaise? Où le train irait-il s'arrêter? Là, il y a eu le souci de se dire qu'on allait demander au conducteur d'actionner le cerclo beaucoup plus souvent, c'est-à-dire toutes les $5 s$ » (Doniol-Shaw \& Foot, 2004, p. 53).

À l'issue de ce séminaire, le responsable de la division tramway du STRMTG (Service Technique des remontées Mécaniques et des Transports Guidés ${ }^{2}$ ), service de l'État en charge de contrôler la sécurité des tramways, synthétise l'accord qui émerge des débats sur le fait que seul le relâchement est symptomatique de la défaillance :

1 Le cerclo est un actionneur de veille situé sous le volant de traction, organe de commande de puissance des trains. Il a donc la forme d'un cercle et le conducteur doit maintenir contre le volant traction en le relevant. En cas de malaise, le maintien du cerclo ne se fait plus et cela initialise la temporisation de 5 secondes au terme de laquelle, sans modification de l'état du cerclo, un freinage d'urgence se déclenche automatiquement.

2 Le Service techniques des remontées mécaniques et des transports guidés a été créé en 2001. Ce service dépend du ministère des Transports et a en charge le contrôle de la conformité des réseaux de tramway et de la sécurité de leur exploitation. 
"Les deux secondes au relâchement correspondent au fait que

l'on considère que, lorsque l'on a une syncope ou un

évanouissement, on relâche »(Doniol-Shaw \& Foot, 2004, p. 68)

Pourtant cet accord ne résiste pas longtemps. Dès le retour à ClermontFerrand, l'autorité organisatrice, maître d'ouvrage du réseau, conseillée par la RATP, demande une contre-expertise pour surseoir à la décision afin, finalement, trois ans plus tard, de refuser toute remise en cause de la vacma.

D'une expertise à l'autre, nous avons poursuivi nos enquêtes sur le travail de conduite dans d'autres réseaux (Clermont-Ferrand, 2009; RATP, 2010 ; Reims, 2011 ; Lille, 2013) et contribué, avec la Fédération National des Syndicats de Transport CGT, à construire un autre réseau d'acteurs, liant syndicats et CHSCT autour de la conduite du tramway. La constitution de cet acteur-réseau s'est faite avec peu de ressources, grâce à la disponibilité de militants et l'action du secrétaire fédéral, Richard Jaubert, pour mobiliser et structurer cet acteur-réseau.

En particulier, trois séminaires, organisés par la FNST-CGT (2011, 2013 et 2016), ont permis de mettre en discussion le métier de la conduite et la conception des cabines de tramway et que se tissent des liens entre syndicats à ce sujet. L'initiative prise par la FNST-CGT de lancer une mobilisation contre la vacma, au plan national en 2012, a été un moment fort d'interpellation par l'acteur-réseau syndical de l'autre acteur-réseau, celui du côté patronal et des services de l'État.

Dans cette dynamique, l'acteur-réseau syndical a su intéresser des services de santé du travail, des experts et des chercheurs. Cette stratégie d'intéressement s'est concrétisée dans un colloque organisé en 2014 (Foot \& Garrigou, 2014). Il faut souligner, qu'un certain nombre de cadres, dans différentes entreprises, ont été également intéressés, parfois explicitement, souvent implicitement, par ces travaux et ont été ouverts aux interrogations sur le travail de conduite que les demandes syndicales ont su poser.

Enfin, dans la dernière période, depuis 2014, nous intervenons avec Laurène Elwert, ergonome dans un cabinet de consultant, dans le cadre d'une assistance à maîtrise d'ouvrage auprès de l'agglomération clermontoise pour la reconception du poste de conduite de son tramway. Dans ce processus, qui devrait déboucher d'ici la fin 2018 sur une refonte complète des postes de conduite, nous avons pu coopérer avec un constructeur et avoir des discussions avec les services de l'État ce qui 
nous a permis de mieux saisir, sinon comprendre, les points de blocage (Elwert \& Foot, 2015).

Cet article s'est nourri, pour la période 2004-2016, de cette dynamique et des données qu'elle a su produire ${ }^{1}$.

\section{La vacma à la $\mathrm{SNCF}$, retour sur recherche pionnière (1963-1972)}

C'est en 1942, d'un point de vue fonctionnel et réglementaire, que les dispositifs de veille se définissent. Le décret ferroviaire du 22 mars 1942, sur la sécurité des systèmes ferroviaires, autorise la conduite à agent unique à condition qu'un dispositif spécial provoque "l'arrêt $d u$ train en cas de défaillance du mécanicien ». Cette défaillance est d'abord pensée dans son cas limite de mort du conducteur d'où le nom d'homme-mort qui servira à désigner ce dispositif de sécurité.

La SNCF confie le soin de penser ce dispositif à deux ingénieurs qui remettront leur rapport en octobre 1942 (Garreau \& Laplaiche, 1942).

Ils définissent les trois conditions principales auxquelles doit répondre ce dispositif :

«1\% d'être infraudable, c'est-à-dire de ne pas pouvoir être paralysé par un moyen à la portée des conducteurs électriciens ;

$2 \%$ d'être inconscient, c'est-à-dire de ne pas exiger du conducteurélectricien une attitude, des gestes ou un effort qui, par leur maintien ou leur répétition, deviendraient une sujétion gênante ;

$3 \%$ d'être efficace en tout circonstance, c'est-à-dire d'entrer en action, non seulement lorsque le conducteur est mort ou évanoui, mais encore lorsqu'il se laisse aller au sommeil » (Garreau \& Laplaiche, 1942, p. 9).

Le rapport conclut qu' « aucun appareil connu ne satisfait parfaitement à toutes » ces conditions. Compte tenu de cette situation, la SNCF, engagée dans l'électrification du réseau et dans la suppression du second agent de conduite, opte à la fin des années 1950 pour un système à appui maintenu que le conducteur peut relâcher pendant 2,5 secondes sans alarme et 2,5 secondes avec alarme sonore avant le déclenchement automatique du freinage. Le caractère fraudable d'un tel dispositif n'est

1 L'ensemble des rapports d'expertise et de recherche est référencé sur le site d'archivage du CNRS HAL-SHS (https://halshs.archives-ouvertes.fr/). Des documents publiés, articles, communications, sont également consultables sur ce même site. Les rapports d'expertises ne sont pas publics mais peuvent faire l'objet d'une demande de consultation auprès des commanditaires de ces expertises (direction et/ou instances de représentations des salariés, $\mathrm{CE}$ et CHSCT). 
pas méconnu mais ce compromis entre la «défiance à l'égard des conducteurs ", qui a orienté vers un dispositif infraudable, et le "scrupule à leur égard», qui a orienté vers un dispositif "ne leur imposant aucune sujétion » (Garreau \& Laplaiche, 1942, p. 14), a trouvé son point d'équilibre dans ce système de « veille automatique » (VA).

Si la SNCF, durant toutes les années 1950, maintient son opposition à l'implantation d'un dispositif de veille automatique avec relâchement périodique qu'elle l'estime «trop astreignante pour le mécanicien »", elle ne s'y opposera plus à partir de 1960 dans le cadre des discussions au sein de l'UIC (Union internationale des chemins de fer) pour unifier les dispositifs d'homme-mort entre les différents réseaux nationaux.

La SNCF, avec l'accélération de l'électrification du réseau et la généralisation de la conduite à un seul agent dans les années 1960, invente alors la vacma (Veille automatique à contrôle de maintien d'appui) pour empêcher la fraude au dispositif de veille, «la Vacma apporte un complément de sécurité par rapport (...) à la VA simple, que le conducteur peut bloquer » (Comité Mixte Professionnel Matériel et Traction, 23/07/65). Cet objectif de lutte contre le blocage volontaire de la veille est explicite dans le cahier des charges réalisé par la SNCF pour développer ce nouvel homme-mort (Ribeill, 1997).

Le nom de ce nouveau dispositif annonce son programme fonctionnel. La Veille Automatique à Contrôle de Maintien d'Appui, la Vacma, a bien pour objet de contrôler la manière dont les conducteurs maintiennent la veille appuyée. La temporisation qui contraint le conducteur à relâcher périodiquement la veille a pour enjeu exclusif de normaliser les comportements par une mise en discipline des corps en rendant la fraude plus coûteuse que l'actionnement temporisé.

\section{La Vacma, naissance du mythe de la « mort crispée »}

Au début des années 1960, un mouvement contre les systèmes de veille, à la fois centralisé par les syndicats et diffus, sous forme d'action directe individuelle, s'est amorcé (Ribeill, 2017). L'introduction de la vacma en 1965 accentue ce mouvement sans toutefois déboucher sur un mouvement collectif de "désobéissance » réglementaire. Wisner, en 1985, rend compte de ce processus d'acceptation du dispositif d'hommemort par les conducteurs : "À l'époque, les conducteurs de train étaient

1 UIC, 5ème Commission, Section Traction, Sous-Commission de circulation des engins moteurs. Paris, février 1960. 
très mécontents de la disparition du deuxième homme dans la cabine. Cela les angoissait. Ils considéraient ce dispositif avec beaucoup d'hostilité. Toutefois, leur attitude s'est progressivement modifiée. À tel point qu'à la fin de notre recherche, les conducteurs nous ont confié qu'ils avaient très bien intégré ce dispositif dans leur activité » (Wisner, 1985, p. 28).

Mais, au début, la Vacma passe mal auprès des conducteurs de train. Des conducteurs CGT demandent, en 1965, à l'équipe de Le Guillant de procéder à une expertise de ce dispositif pour suppléer le mouvement de lutte contre la Vacma (CGT, 1966; Moscovitz, 1970; Le Guillant et al., 2006; Couëdel \& Nouailhetas, 2017).

De manière inattendue, l'hypothèse fantasmatique de la " mort crispée », qui n'était pas évoquée en 1942, apparaît d'abord de manière marginale du côté de la direction tandis qu'elle s'affirme du côté des conducteurs et des syndicats.

Du côté de la direction, cette expression reste, en effet, secondaire. On trouve trace de cette " défaillance crispée », par exemple, dans un texte d'un service technique où l'idée "d'une défaillance du conducteur qui n'entraînerait pas le lâchage de l'appui ${ }^{1}$ est évoquée. Il faut se tourner du côté syndical pour voir cette hypothèse explicitée et socialisée au travers de tracts et de publications.

Ainsi, dès 1963, on trouve trace dans les écrits syndicaux de l'idée de «mort crispée» à propos de la VA : "On retrouvera le conducteur mort, les mains crispées sur le levier, ou le genou coincé sur la pédale $d$ 'homme mort $\|^{2}$. Mais c'est avec la lutte contre la vacma que cette idée s'affirme et devient plus consistante, que cette hypothèse va se renforcer et devenir explicite, «prendre corps » pourrait-on dire, au travers de l'avis médical donné par l'équipe de médecins.

\section{L'ambivalence de la critique syndicale de la vacma}

Du côté syndical, l'idée d'une «mort crispée » se diffuse et se formalise de manière explicite, en lien avec l'émergence de l'électricité dans l'exploitation des trains. L'électrocution apparait alors comme un danger nouveau auquel est confronté le groupe conducteur. Ce danger est essentiellement concentré autour des interventions en toiture dans cette

1 Annexe à la lettre ML8L3 n ${ }^{\circ}$ 178, signé par le Chef de la Division de l'entretien des Engins Moteurs le 23 juillet et approuvé par le Chef du Service du Matériel et de la Traction le 11 août 1965

2 CFTC, Bulletin de liaison roulants Sud-Ouest, $\mathrm{n}^{\circ}$ 10, 1er avril 1962 (Ribeill, 2017). 
période de transition entre le charbon et l'électricité (Couëdel \& Nouailhetas, 2017), pourtant le lien est fait avec la conduite bien que, dans la cabine, le risque d'électrocution n'existe pas.

Le dispositif d'homme-mort, associé à l'électrification du réseau et à la suppression de l'aide-conducteur, apparaît être un opérateur qui introduit le danger " électrique » dans le poste de conduite ${ }^{1}$. Dans ce processus, cet homme-mort se charge d'une puissance symbolique qui étend le danger de mort à l'ensemble de l'activité des conducteurs.

Le texte de la conférence de 1966 sur les conditions de travail des agents de conduite condense ces positions de la CGT et donne corps à l'existence d'un malaise-crispé voire d'une «mort crispée» (CGT, 1966).

L'hypothèse de la «défaillance crispée » est convoquée à plusieurs reprises tant à propos de la VA :

«En cas d'évanouissement, le conducteur peut coincer la pédale ou se crisper sur le cerclo (cas d'électrocution et le train continue sa marche sans que rien ne s'y oppose) $»^{2}$.

que de la Vacma:

«Le conducteur d'un express (à vitesse limite $140 \mathrm{~km} / \mathrm{h}$ ) roulant à 110 $120 \mathrm{~km} / \mathrm{h}$ franchit un signal jaune. Il a préalablement réarmé la Vacma pour se libérer de cette préoccupation, il arrête le klaxon dont la mise sous tension a été provoquée par le franchissement du crocodile au signal jaune. Il est pris de malaise, s'effondre, coince la pédale ou se crispe sur le cerclo (...) Les 55" épuisées, la sonnerie retentira, le conducteur ne réagira pas et la Vacma fonctionnera seulement à ce moment-là (donc une minute). Il faudra ajouter la distance de freinage 700 à $800 \mathrm{~m}$ et le convoi marquera l'arrêt. Donc au total $2 \mathrm{~km} 800$. Lorsque l'on sait qu'immédiatement derrière le signal d'arrêt peut se trouver la queue du train précédent... » ${ }^{3}$.

Cette critique de la VA, ou de la Vacma, est ambivalente car le principal reproche fait à la Vacma et, a fortiori à la VA, est une temporisation trop longue qui ne permettrait pas de détecter une "défaillance crispée » à temps. Progressivement, la fonction de la vacma se redéfinit d'un contrôle de la fraude à celle d'une détection d'une "défaillance crispée $\gg$ :

«La VA, ou veille automatique (...) fut améliorée car d'une part on

1 Dans le métro parisien, ce rapport à l'électricité et à ses dangers a structuré toute une symbolique institutionnelle tant au niveau de la direction que du syndicat Traction (Foot, 1991).

2 (CGT, 1966, pp., p.12.)

3 (CGT, 1966, pp., p.12.) 
pouvait facilement le bloquer avec une ficelle et, d'autre part, si le conducteur se crispait sur le volant à la suite d'une syncope, la sécurité ne fonctionnait pas. On la remplaça donc par la VACMA » (Techniques et méthodes de l'exploitation capitaliste. Enquête médicale auprès des roulants. L'esclavage de la vacma. Cahier de Mai, 1970, n²1, p.16).

Dès lors que la «défaillance crispée » devient un danger « réel » aux yeux des syndicalistes, que le fantasme prend le pas sur le réel physiologique, la lutte contre la vacma perd de sa légitimité. Paradoxe d'une lutte contre la Vacma qui finit par, sinon militer, du moins préparer l'acceptation d'une augmentation de la sujétion du conducteur alors que le combat pour le second agent s'estompe.

Dans le même temps où la fiction d'une "défaillance crispée » prend pied dans le réel, où cette fiction devient un « réel», une autre réalité sociale, pourtant bien effective celle-là, la fraude à la veille, le calage ou l'isolement, est rejetée dans le domaine de la fiction :

«Dernier argument mis en avant par la SNCF pour substituer la Vacma à la VA: "La mise en place de la Vacma a été rendue nécessaire du fait que les conducteurs trichaient avec la VA simple en attachant le cerclo au volant".

Argument qu'aucun agent de conduite ne peut accepter.

La sécurité pour tous les cheminots est un souci majeur, aussi les roulants rejettent de tels artifices, ils veulent lutter contre le système, pour son abolition et non pour le dénaturer ${ }^{1}$

Ce déni de la fraude, justification première à l'implantation de la vacma, n'est pas le seul fait des syndicats. On constate aussi, du côté des directions, que cet argument est peu mobilisé pour la justifier dans les discussions avec les syndicalistes. Entre déni et effacement, la fraude laisse ainsi progressivement toute la place à la "défaillance crispée " pour justifier de ce dispositif.

Progressivement, la question de la vacma va s'effacer de la scène sociale. La période ouverte par Mai 68 avec la montée d'une issue politique aux luttes sociales liées au programme commun ne sera pas favorable aux luttes catégorielles dont la vacma est un exemple typique. Cette nouvelle conjoncture associée aux difficultés d'une mobilisation efficace contre la vacma amène les syndicalistes à composer avec ce dispositif $^{2}$.

1 (CGT, 1966, pp., p.12.)

2 On peut suivre cette évolution dans les comptes rendus des Comités Mixtes Professionnels Matériel et Traction, de 1963 à 1973. 


\section{La légitimation de la « défaillance crispée » par les médecins}

Le recours à des psychiatres, à la science, en 1965, apparaît être «le moyen légal de supprimer un appareil de sécurité » (Moscovitz, 1972, p. 216) susceptible de se substituer au rapport de force quand celui-ci ne parvient pas à se constituer autour de cette question. Cette équipe de médecins n'interroge pas la fonction de cet homme-mort. Ces médecins vont reprendre, en l'état, les liens fonctionnels établis par les conducteurs entre le dispositif de veille et la défaillance ${ }^{1}$.

Il est extrêmement troublant qu'ils se saisissent ainsi de cette idée de malaise-crispé comme justification technique de la vacma, repoussant également sa justification comme dispositif anti-fraude au motif que cette fraude serait «tout à fait exceptionnelle » :

"Cependant, la chute ou la crispation sur le cerclo en cas de malaise, ou sa fixation par certains procédés - qui a été avancé mais paraît tout à fait exceptionnelle - pourraient compromettre son jeu "automatique" - et la sécurité. D'où l'introduction de la vacma » (Le Guillant et al., 2006, p. 151).

Le texte publié par Moscovitz en 1972 reprend le même argumentaire, en précisant même que la crispation pouvait provenir d'un «cas de malaise, ou de syncope ». La fraude, par contre, n'est plus minorée, n'est plus renvoyée à des situations d'exception :

"Cependant la chute ou la crispation sur le cerclo en cas de malaise, ou de syncope, ou une possible fixation volontaire par certains procédés, pouvaient compromettre son jeu automatique, et donc la sécurité du convoi - d'où l'introduction d'un système identique mais plus perfectionné : la Vacma » (Moscovitz, 1972, p. 188).

Les médecins reprennent à leur compte le point de vue développé spontanément par les conducteurs :

«Je redoute le malaise cardiaque, mortel, immédiat, qui me crisperait sur la veille automatique» (Réponse d'un conducteur à l'enquête par questionnaire. Moscovitz, 1970)

Or s'il y a une chose de médicalement établie, c'est bien le fait qu'il n'existe pas de « défaillance crispée » :

"Subitement privé de la vie, le corps s'effondre, inerte, plaqué au sol par les forces de la pesanteur. Les muscles sont mous, et les pièces mobiles du squelette peuvent être mises dans toutes les positions mécaniquement possibles.

Coupé de ses relations avec le monde extérieur, l'organisme anesthésié ou endormi présente la même immobilité, une pareille mollesse de la musculature, une semblable passivité »(Paillard, 1976, p. 521).

1 L'analyse faite de cette intervention dans le présent article ne porte que sur leur rapport à la Vacma et au malaise-crispé. Sur la question de l'apport de cette intervention à la psychologie du travail, en particulier sur l'introduction de concepts psychanalytique, on pourra se reporter utilement à l'analyse faite par I. Billiard (Billiard, 2001, pp. 244-249) 


\section{Déni du corps et de la technique dans l'approche psychiatrique}

Cette légitimation du «malaise-crispé » apparaît être la conséquence d'une sorte de double déni que se pose ce groupe de médecins dans leur approche des conditions de travail. C'est d'abord le refus de considérer l'objet technique «vacma» et d'analyser sa fonction. En effet, les médecins de 1965 ne se considèrent pas «qualifiés pour (...) juger» de cette mise en sécurité avec la vacma (Le Guillant et al., 2006, p. 151). Pourtant, et Moscovitz le précise dans son texte, il ne s'agit pas d'abord d'un système adressé au train mais d'un outil de diagnostic pour détecter la défaillance du conducteur :

"Ce dispositif permettrait une sécurité accrue : nous ne sommes évidemment pas habilités à émettre notre avis en matière de sécurité des convois Sncf. En fait, cette sécurité est recherchée en cas de défaillance du conducteur. Nous disons bien $d u$ conducteur » (Moscovitz, 1972, p. 188).

Ce refus d'une prise en compte de la fonction technique de la vacma ne se limite pas au diagnostic de la défaillance, mais s'étend à l'ensemble des fonctions techniques associées à la vacma :

«nous ne nous étendrons pas sur les autres aspects techniques de la vacma » (Le Guillant et al., 2006, p. 151).

Ce premier déni technique se double alors d'un autre refus de prise en compte du réel, celui du corps dans la subjectivité. Ce déni du corps s'articule au travail de distinction qu'accomplit ce groupe de médecins, non seulement pour faire reconnaître la spécificité d'une approche psychiatrique des conditions de travail, orientée vers la psychanalyse par Moscovitz (Billiard, 2001, pp. 244-249). Dans cette perspective, il leur apparaît important que leur démarche psychiatrique ne puisse être confondue ni avec «une approche neuro-physiologique» ni avec celle «du Médecin du Travail » (Moscovitz, 1972, pp. 186-187; 191).

Ainsi, censurant leur compétence médicale tant pour l'analyse d'un outil de diagnostic «médical » que pour la définition physiologique de la défaillance, et afin de privilégier "une compréhension psychopathologique du problème » (Moscovitz, 1972, p. 187) posée par la vacma aux conducteurs, le psychiatre habilite alors le milieu ferroviaire d'une compétence à concevoir cet outil de diagnostic et de définir la forme prise par la défaillance. Que cette définition du dispositif d'Homme-mort autour de la «mort crispée » soit absurde, échappe, par construction épistémologique, au point de vue de ces psychiatres. Ce n'est pas, pour eux, un objet de questionnement. 
Une fois le monde du travail «purifié » de sa matérialité tant technique que biologique, l'analyste est amené à privilégier " l'histoire personnelle du sujet » et «Le psychiatre doit avant tout mettre l'accent sur l'Homme et sur son vécu personnel » (Moscovitz, 1972, p. 192).

À cette dématérialisation de l'espace de travail se substitue sa subjectivation au point que l'émergence de la mort dans les discours des conducteurs est renvoyée à n'être l'expression que de "phantasmes de castration: thème de folie ou de mort, ou thèmes de catastrophes ferroviaires imminentes » (Moscovitz, 1972, p. 204).

La réalité de cet «homme-mort» devenu «vacma », l'histoire personnelle de cet objet technique dont la fonction primaire était de demander au conducteur de dire à la machine s'il est encore vivant puis, dans sa version vacma, de le contraindre à ne pas frauder, s'est effacée de l'analyse psychanalytique. La manière dont cet objet s'est transformé, s'est rempli d'une nouvelle fonction avec la temporisation au maintien, i.e. l'obligation périodique de relâcher, celle de détecter une mort fantasmée, la « mort crispée », qui a pris la place du contrôle anti-fraude n'a pas été analysée. Ce changement de sens du geste n'a pas fait sens dans cette approche psychiatrique.

Cet effacement de l'objet se lit également dans l'absence de «l'hommemort » dans les différents textes produits au cours de ces recherches. Ce terme qui définissait crûment la fonction de ce dispositif avait été «neutralisé », désaffecté, par l'UIC (Union Internationale des Chemins de fer) en 1961 car cette "désignation [...] devrait disparaître, eu égard aux réactions possibles $d u$ grand public, du personnel etc. ${ }^{1}$. Le terme de vacma correspond à l'invention de cette "règle de langage» dans le milieu ferroviaire qui permet d'éviter que le rapport direct d'une chose à sa fonction soit explicite quand ce rapport exprime une violence potentiellement déstabilisatrice pour l'action (Arendt, 1991, pp. 144-145). Dans les différents textes produits lors de cette recherche, seule la forme " neutre » de vacma qui désaffecte le terme de sa puissance est mobilisée. Il est à noter que, au contraire, Wisner qui a mené une recherche en parallèle à la SNCF, rappelle que «la question posée initialement concernait "l'homme mort" "(Wisner, 1985, p. 28).

1 L'UIC décide, à l'initiative de la Deutsche Bahn, de changer la dénomination de ce dispositif : "La désignation "Totmann”-Vorrichtung devrait disparaître, eu égard aux réactions possibles du grand public, du personnel etc. et être remplacée dans le texte allemand par "Sicherheitsfahrschaltung". Il restera à examiner si, dans le texte français, une autre expression pourrait remplacer "homme-mort" " UIC, 5 ème Commission, Section Traction. Sous-commission de circulation des engins moteurs. Février 1961. 
Si l'on substitue au terme de vacma celui d'homme-mort, il apparaît moins nécessaire de recourir aux "phantasmes de castration" pour comprendre la présence dans les discours des conducteurs des "thème de folie ou de mort, ou thèmes de catastrophes ferroviaires imminentes ». Ce dispositif propose précisément un «script»d'action pour prévenir des «scénarios » possibles (Akrich, 1987), la mort d'un conducteur entraînant une catastrophe ferroviaire étant le scénario de référence. Comme dispositif de sécurité ultime, l'homme-mort exprime la fonction principale de ce dispositif, détecter la mort, tandis que la vacma, le contrôle du mode de maintien d'appui, cadre, discipline l'interaction du conducteur et de ce dispositif, afin de prévenir un débordement potentiel, une fraude au dispositif qui se traduirait par son blocage volontaire en position maintenue à l'aide d'un artefact quelconque (Latour, 1994).

Le conducteur est ainsi amené à se confronter à sa propre mort au moins toutes les minutes, à « dire » par geste à la machine qu'il est toujours vivant, pour l'instant. Il y a un rappel incessant que la mort est constamment présente et que le système de machine doit, le premier, être averti de son advenue. La violence de cette confrontation dans le travail à la mort, à sa propre mort, est d'autant plus forte que, dans le même temps, dans la société, les morts "sont escamotés» (Thomas, 1980). Il n'est dès lors guère étonnant que cette violence du rapport à la mort s'exprime dans les entretiens menés par Moscovitz:

"Oui, c'est le cour. Chaque fois que je prends une machine avec vacma, j'ai un pincement au cœur [...] Avec tous ces petits chocs, le cæur, les nerfs que deviennent-ils? C'est comme quand une voiture surgit brusquement devant vous. Eh bien moi, ça me fait ça toutes les cinquantecinq secondes » (Moscovitz, 1970, p. 510).

La piste psychanalytique proposée par Moscovitz pour sortir de l'impasse dans laquelle la recherche sur la vacma semble être (Le Guillant et al., 2006, p. 163) n'apparait pas convaincante pour comprendre la fonction de la vacma dans les réactions des conducteurs. Bien que cet objet singulier inscrive explicitement la mort au cœur de l'activité de conduite et oblige le conducteur à lui " dire » qu'il est (encore) vivant, la thématique de la mort, qui apparaît dans les discours des conducteurs, est complètement détachée du dispositif d'Hommemort, de son principe fonctionnel pour être rattachée exclusivement à l'histoire des sujets. Le primat donné à leur histoire personnelle empêche alors de saisir la dimension anthropologique, impersonnelle, autour de la mort et de la solitude au travail dans les mondes industriels modernes, de la vacma. 
Cette absence de réflexion sur la représentation de la mort incarnée par ce dispositif qui se double d'un refus de considérer la «technique du corps défaillant » ne permet pas de donner prise à une déconstruction de ce mythe émergeant dans le monde ferroviaire, celui de la «mort crispée $»$.

\section{D'un fantasme à l'autre, de la « mort crispée » au contrôle de la vigilance}

La capacité du dispositif d'homme-mort à acquérir une fonction fantasmatique, la traque d'une "défaillance crispée », en déconnexion avec toute réalité physiologique, lui a donné probablement une compétence à agréger d'autres fonctions toutes aussi illusoires. Ainsi en va-t-il pour le contrôle de vigilance.

Bien que dès le début de l'introduction de la vacma, Wisner avait constaté que les conducteurs avaient "très bien intégré ce dispositif dans leur activité, mais que son usage correct ne les empêchait nullement de s'assoupir en conduisant »(Wisner, 1985, p. 28), cela n'empêche pas que progressivement l'on passe d'une définition fonctionnelle de la vacma, comme dispositif de diagnostic de la défaillance, à celle d'un système de contrôle de la vigilance.

Ainsi, dans la réunion du Comité Mixte Professionnel Transport \& Commercial du 14 mars 1973, il est fait état des "fautes de vigilance » pour les cinq derniers exercices entre 1967 et 1972 pour désigner les freinages d'urgence dus au défaut d'actionnement de la veille.

La définition fonctionnelle de la vacma avec son l'obligation périodique de relâcher s'enrichit donc. Alors que sa fonction "anti-fraude » s'estompe, le contrôle de la vigilance vient ajouter un nouveau rôle à la vacma en plus de la détection du malaise. Cette nouvelle fonction autorise alors la SNCF à valoriser ce dispositif au nom de la sécurité, valeur centrale dans la corporation ferroviaire, valeur partagée par les conducteurs ${ }^{1}$.

1 Cette substitution de la fonction vigilance à celle du contrôle anti-fraude pour caractériser le relâchement périodique de la veille n'est pas spécifique à la France, on la retrouve, par exemple, dans le milieu ferroviaire britannique : "Un système plus sophistiqué fut conçu dans les années 60, habituellement défini comme dispositif de sécurité pour le conducteur ou de contrôle de vigilance. Son fonctionnement suppose que le conducteur manifeste sa vigilance en actionnant périodiquement un bouton du pupitre de commande ou en appuyant sur une pédale spécifique ", in Simmons Jack \& Biddle Gordon (eds.), 1997, The Oxford companion to British railway history, from 1603 to the 1990s, Oxford University Press, p.125. 


\section{La VACMA et le tramway, l'histoire moderne de fantasmes têtus (1985-2017)}

En 1985, avant que le tram ne soit réintroduit en France, les systèmes de veille dans le ferroviaire urbain n'ont guère suscité d'intérêt. À SaintÉtienne et Marseille, les tramways sont équipés d'un système de veille automatique sans contrainte de relâchement et à Lille, il n'y a, depuis 1984, plus aucun système de veille. Seule, la RATP au début des années 1970, a été amené à réfléchir à l'introduction d'un système d'hommemort sur les matériels roulants modernes du métro. Cette introduction est liée à la conduite du métro avec un agent « seul à bord du train » depuis que le chef de train est progressivement retiré au fur et à mesure du renouvellement des rames et du retrait des « spragues ».

$\mathrm{Au}$ moment de la réintroduction du tramway en France, la RATP se trouve être le seul exploitant ayant des compétences en la matière même si à la même période, le service des Facteurs Humains de la SNCF s'interroge sur la pertinence de leurs systèmes de veille. Mais ces études ne déborderont pas du périmètre d'influence de ce service et, de plus, seront arrêtées sans être parvenues à déboucher sur un résultat concret (Vignes in Doniol-Shaw \& Foot, 2004, pp. 42-44).

C'est donc assez naturellement que la RATP se trouve être un opérateur central de la conception des dispositifs de veille dans ce retour du tramway en France, à partir de 1985. Dans ce processus, qui ne tiendra aucun compte des recherches effectuées à la SNCF ou ailleurs, la vacma s'imposera sur un mode frénétique puisque le temps de maintien maximum autorisé de la veille sera de l'ordre de dix secondes.

Il faudra plus de vingt ans pour qu'un débat remettant en cause l'assise fantasmatique de la vacma, tant sur la dimension vigilance que sur celle de la «mort crispée» ait lieu. Mais la seule ouverture d'un débat rationnel ne suffit pas à changer les choses. L'entrée dans ce débat de la Fédération des transports CGT en 2012, associée à l'impulsion d'une action contre la vacma dans les réseaux, donnera une force nouvelle, un caractère plus performatif, à cette remise en cause de la vacma.

Le contrôle de vigilance, un fantasme qui résiste depuis plus de dix ans aux épreuves de la science et de l'expérience

Cette fiction d'une vacma qui assurerait au-delà du diagnostic de défaillance, un contrôle de vigilance n'a pas été interrogée par la SNCF jusqu'au début des années 1980 où, du fait de l'informatisation 
croissante des postes de conduite, des interrogations ont émergé sur les possibles améliorations des systèmes de veille. Des recherches ont été menées qui ont conclu :

«Il convient de souligner que le système Vacma, à l'origine, était destiné à déceler les pertes de conscience éventuelles du conducteur, sans que sa vigilance, en conduite normale, fasse l'objet d'une surveillance particulière par ce système. (...) Il a également été constaté pendant ces phases de vigilance atténuée, un accroissement de la régularité dans les relâchés de la pédale et une augmentation de la durée du relâchement de l'appui. Aucun des agents, au cours des 80 expérimentations n'a eu conscience de ces périodes d'hypovigilance qui sont restés transitoires, le sujet redevenant ensuite totalement vigilant. La périodicité de ces phases de moindre vigilance est comprise entre 90 minutes et 120 minutes et elles peuvent durer 30 minutes » (Mollard et al., 1991, pp. 66-67).

Une étude similaire menée en Allemagne avec l'équivalent de la Vacma pour ce pays, le système SIFA (Sicherheitsfahrschaltung) aboutit à des conclusions similaires :

"Le dispositif expérimental que nous avons développé a réussi à faire baisser le niveau de vigilance des sujets jusqu'à ce que leurs réactions à des événements imprévus soient improbables. Néanmoins, le SIFA continue à être exploité normalement, c'est-à-dire sans que la baisse de vigilance n'ait pour conséquence de provoquer un freinage d'urgence comme attendu dans les conditions réelles de conduite. Nos résultats, obtenus dans des conditions de laboratoire, confirment les résultats tirés de l'observation et des études préliminaires » (Peter et al., 1983, p. 337).

Outre ses connaissances scientifiques, une enquête diligentée par le BEA $\mathrm{TT}^{1}$, suite à un tamponnement entre deux rames survenu le 30 août 2004 sur le réseau de Rouen, conclut que le conducteur de la rame qui a percuté la rame précédente était dans une phase de « pré-sommeil »:

"On peut conclure de l'absence de toute réaction du conducteur qu'il est alors entré en phase de pré-sommeil avec occlusion des paupières, mais maintien d'un agissement musculaire sur le manipulateur avant d'entrer en phase de relâchement. L'accident a précisément lieu à cet instant » (BEA-TT, 2005, p. 36).

Pas plus que les connaissances physiologiques n'ont empêché le milieu ferroviaire de produire la fiction de la «mort crispée», ces connaissances, issues tant du milieu de la recherche que du milieu ferroviaire, sur l'incapacité de la vacma à détecter les états d'hypovigilance, voire d'assoupissement, n'ont pas plus empêché ce même milieu ferroviaire d'attribuer une nouvelle fonction, fictionnelle elle aussi, à la vacma, celle de contrôler la vigilance.

1 Le Bureau d'Enquêtes sur les Accidents dans les Transports Terrestres a été créé en 2004. Il est un service à compétence nationale. 
L'exemple le plus caractéristique d'un tel processus de refoulement de la connaissance de la réalité fonctionnelle de la veille, nous est donné par le STRMTG. En 2004, lors du colloque organisé par T2C et le Latts, le responsable de la division tramway affirmait :

"La VACMA et la vigilance? On l'a vu tout au long de la journée, et nous en sommes persuadés, la VACMA n'est pas un outil de vérification de la vigilance. C'est un outil pour dire si le conducteur est présent à son poste ou s'il ne l'est pas. Cela ne va pas plus loin» (Arras in Doniol-Shaw \& Foot, 2004, p. 61).

Pourtant, quand, huit ans plus tard, le STRMTG publie son guide d'ergonomie sous la direction de ce même responsable, cette affirmation est oubliée. La veille y est définie comme "un dispositif permettant de s'assurer de la vigilance du conducteur » (STRMTG, 2012, p. 12).

Le terme de " vigilance » est utilisé huit fois, il sert même à désigner le dispositif lui-même, "la commande vigilance », tandis que le terme de défaillance, terme utilisé dans le décret de 1942, n'est nullement mentionné.

Il faut attendre 2015, et la publication d'une troisième version de ce guide, pour que ce terme de vigilance disparaisse et que soit restauré la fonction initiale du dispositif de veille, celle de la détection de «la perte de connaissance ». Ce n'est plus la vigilance du conducteur qui est évaluée mais le fait d'être «conscient» (STRMTG, 2015, pp. 16-17).

Il aura donc fallu plus de dix ans pour que les connaissances scientifiques ainsi que les leçons de l'accident de Rouen produites et discutées en 2004 soient appropriées et formalisées par le milieu du tramway. Probablement, la résistance de ce fantasme est corrélée également à la difficulté éprouvée par les responsables de «vendre » cette vacma aux conducteurs si la seule justification à l'imposition de cette contrainte est de prévenir la fraude du système. La vigilance serait aussi un « élément de langage » dans la négociation avec les conducteurs qui, progressivement, finit par acquérir le statut de qualité intrinsèque du dispositif alors que s'estompe sa fonction anti-fraude.

Cette occultation de la fonction « anti-fraude » est toujours présente dans les manières de justifier l'obligation de relâchement en station quand est publié le guide «Fonction veille » en 2017. Le STRMTG n'évoque pas cette fonction anti-fraude quand il prescrit une obligation pour le conducteur de procéder " à un cycle d'appui-relâchement» pour pouvoir partir d'une station. Le guide justifie cette obligation en inventant une novlangue ferroviaire qui fait de la lutte contre la fraude une action "pour que le conducteur soit toujours conscient de l'existence d'une fonction veille». 
La peur de la « mort crispée » accélère le tempo de la vacma des tramways

Dans le développement de systèmes de veille pour les tramways, la RATP intègre explicitement le fantasme de la "mort crispée ». Les concepteurs s'interrogent, en effet, sur la temporisation à retenir en cas d'exploitation en mode dégradé :

"On s'est également demandé ce qui pourrait se passer si l'arrêt automatique ne fonctionnait pas, s'il était hors service par exemple, et que le conducteur avait un malaise? Où le train irait-il s'arrêter? Là, il y a eu le souci de se dire qu'on allait demander au conducteur d'actionner le cerclo beaucoup plus souvent, c'est-à-dire toutes les 5 s, si bien qu'un conducteur ayant un malaise, avec un arrêt automatique hors service, et franchissant un signal fermé, le train s'immobiliserait au bout de $5 \mathrm{~s}$, puisqu'il faut actionner le système toutes les $5 s$ » (Caligny, responsable des études fonctionnelles matériel roulant, RATP, in Doniol-Shaw \& Foot, 2004, p. 53).

La crainte du malaise-crispé est toujours présente même si, dans les représentations, l'électrocution a laissé place depuis longtemps à la crise cardiaque d'autant plus que, depuis les années 1960, les risques d'électrocution ont disparu des postes de conduite. L'accident de Rouen du 30 août 2004 met en scène cette substitution :

"Un feu a donc été franchi par un conducteur, qui a fait un malaise, estce qu'il était cardiaque ou autre, ça on ne le sait pas encore».

"Mais le conducteur peut avoir une crise cardiaque et s'appuyer sur son manipulateur et là il reste appuyé et alors le temps de maintien est important ? " (Conducteur, CHSCT Rouen, in Doniol-Shaw \& Foot, 2004, p. 57).

La temporisation maximum de maintien est un indicateur de cette crainte d'une défaillance ou d'une «mort crispée ». Pour les concepteurs des systèmes de veille, un malaise peut aussi bien se traduire par une crispation que par un relâchement. Il faut alors, par conséquent, obliger le conducteur à lâcher prise pour qu'il puisse prouver qu'il est bien là, conscient. Le relâchement qui sert initialement à prouver que le système n'est pas fraudé est devenu maintenant aussi le moyen de prouver qu'il n'est pas mort !

Cette construction fantasmatique se cristallise dans les dispositifs de veille des tramways où l'environnement urbain sert à justifier cette accélération du rythme d'action sur la veille, du rythme de l'alternance du maintien et du relâchement :

"Sur les tramways, ce sont des cycles de $15 \mathrm{~s}$ avec $13 \mathrm{~s}$ de maintien et $2 \mathrm{~s}$ de relâchement. (...). On voit bien aujourd'hui, qu'en fonction de l'environnement dans lequel circule le matériel roulant, les temporisations sont différentes. Sur un tramway en milieu urbain, en $15 \mathrm{~s}$, il peut se 
passer beaucoup de choses. Le conducteur circule, en milieu ouvert, et cette temporisation de $15 \mathrm{~s}$ semble appropriée. (...)

On a des cycles plus courts, parce qu'on est dans un milieu urbain, un milieu ouvert, pas complètement maîtrisé» (Mons, représentant Mode Tram, RATP, in Doniol-Shaw \& Foot, 2004, p. 52; 56).

Quand le tramway est réintroduit en France à partir de 1985, c'est la conception d'une veille en charge de traquer aussi bien les défaillances relâchées que crispées qui l'accompagne. Mis à part Nantes, tous les autres réseaux auront une vacma temporisée sur un cycle d'environ $10 \mathrm{~s} / 2,5 \mathrm{~s}$. Probablement, parce que le milieu du tramway est en train d'émerger et qu'il n'est donc pas encore structuré, les conducteurs de Nantes sont parvenus à refuser ce dispositif. Une veille automatique, i.e. sans obligation de relâcher périodiquement la veille, se substitue alors, pour ce premier tramway moderne, au projet de vacma (Doniol-Shaw \& Foot, 2004, pp. 11-12).

Ce parti-pris technique s'impose sans explication. Nulle part, y compris à la RATP, on ne peut trouver une justification fonctionnelle explicite. Lors d'une expertise réalisée en 2010, le directeur délégué au tramway nous confirmera "qu'il n'a pas trouvé de justification formalisée à la vacma et sa temporisation » et "énonce que le maintien de la vacma sur les nouvelles lignes correspond au souci des responsables de maintenir une homogénéité organisationnelle » (Doniol-Shaw et al., 2010). Naturellement, aucune étude ne sera faite.

La crainte d'un «malaise-crispé » même si elle n'est jamais véritablement explicitée est néanmoins présente dans le milieu du tramway comme en témoigne un rapport d'enquête du BEA-TT :

"Il existe ainsi un dispositif usuellement dénommé "veille ", que le conducteur doit actionner périodiquement. S'il oublie de l'actionner ou s'il l'actionne continûment, un freinage d'urgence est automatiquement déclenché, en considérant que le conducteur pourrait avoir fait un malaise »(BEA-TT, 2011, p. 20).

Cette même interprétation est reconduite dans le rapport du BEA TT sur l'accident de Montpellier survenu cinq ans plus tard. Bien que centré sur le rôle de la vacma dans un accident qui a causé la mort d'un voyageur, ce rapport manifeste toujours la même ambigüité du milieu à la vacma :

"On peut par ailleurs s'étonner des caractéristiques techniques des dispositifs actuels de veille de type VACMA équipant majoritairement les rames françaises de tramway, qui recourent à une action fréquemment répétée d'un conducteur à la seule fin que les automatismes ne le considèrent pas victime d'un malaise ou décédé " (Souligné par nous, BEA-TT, 2016, p. 40). 
Cet étonnement ne se traduit pas par une critique de cette logique où la répétition fréquente des actions, qui n'a pas de sens d'un point de vue fonctionnel, est considérée comme un mode pertinent de détection de la défaillance. Aucune recommandation émise par le BEA-TT ne vise à proscrire cette logique irrationnelle de temporisation.

Il faut attendre 2017 pour que, enfin, le STRMTG déclare officiellement : "les malaises conduisant à une crispation des membres ne sont plus pris en compte » (Souligné par nous, STRMTG, 2017, p. 7). À rebours, cela signifie que, jusque-là, c'est bien le fantasme du malaisecrispé qui hantait les responsables et imposait sa frénésie aux conducteurs.

\section{L'action syndicale et le difficile retour au réel du milieu tramway (2000-2017)}

Cette déconstruction du rapport fantasmé des responsables du milieu tramway au dispositif d'homme-mort, tout elliptique qu'elle soit, est néanmoins réelle. Elle est l'aboutissement, encore inachevé, d'un processus complexe où l'action syndicale joue un rôle central.

Une partie de la complexité de ce processus tient au fait que le milieu du tramway est un vrai milieu et que, pour une part, l'action syndicale ne lui est pas extérieure. Pour agir, il faut en faire partie mais ne pas être complétement «pris» par ce milieu (Favret-Saada, 1985). Il faut pouvoir s'en déprendre suffisamment pour remettre en question les évidences partagées, comme celui de la «mort crispée» ou de la fonction de contrôle de vigilance de la vacma. Mais la modalité d'incorporation de ces évidences, distribuées dans chaque manipulateur de tramway, est redoutable. Elle discipline le corps de chaque conducteur et force chacun à considérer sa conduite comme rationnelle et par conséquent à admettre la rationalité de ce dispositif. Si on ne l'accepte pas, alors, poursuivre la conduite du tramway deviendrait insupportable.

La subjectivité du rapport au travail s'inscrit dans ce rapport au corps, d' " un corps qui est un Je » pour reprendre la belle expression de M. Henry cité par Molinier (Molinier, 2008, p. 62).

La force persuasive de la vacma réside en ce qu'elle marque chacun dans son corps en même temps qu'elle constitue un marqueur symbolique de son entrée dans le monde du tramway. Cette identification, quasi 
symbiotique, entre la vacma et le conducteur se manifeste concrètement dans le fait que, au début de l'entrée dans le métier, actionner la vacma et conduire le tramway ne font qu'une seule et même chose. Ne pas faire sonner l'alarme veille c'est la preuve d'une maîtrise de la conduite.

Un accident de tramway manifeste cet état des relations entre les conducteurs et la vacma. Un conducteur de bus qui vient d'être habilité à la conduite du tramway depuis trois jours, stoppe son tramway à un signal fermé dans une rampe à $6 \%$. Au moment de repartir, le conducteur ne tractionne pas suffisamment et part en dérive. Cette dérive en marche arrière dure 21 secondes durant lesquelles le conducteur essaie de tractionner pour arrêter la dérive mais celle-ci se poursuit et le tramway accélère jusqu'au choc contre une autre rame à la vitesse de $26 \mathrm{~km} / \mathrm{h}$. Durant tout ce temps, où ce conducteur essaie de rattraper cette dérive, il ne pense pas plus à freiner qu'à lâcher la veille. Il tractionne et veille constamment. Ce n'est qu'après le choc qu'il lâche la veille et que le freinage d'urgence se déclenche (BEA-TT, 2011).

Ce conducteur débutant manifeste, dans une montée aux limites, comment la formation à la conduite du tramway désapprend les comportements ordinaires, comme freiner pour éviter un accident, et, par contre, apprend à se focaliser sur le manipulateur et la vacma. À l'issue de cette formation, avant que le métier ne s'acquière, la vacma est devenue prééminente dans la conduite. Son actionnement a pris le pas sur l'efficacité de la conduite. La dérive du tramway manifeste la dérive $\mathrm{du}$ réel du système de conduite. Cet apprenti conducteur traduit, au premier degré, la folie de ce système où les fonctions fantasmatiques l'ont emporté sur la fonction sécuritaire première. Si ce conducteur avait arrêté de veiller, le tramway se serait arrêté également mais pour lui, à ce moment-là, arrêter de veiller aurait signifié arrêter d'être conducteur. Il poursuit sa conduite jusqu'à l'accident.

L'intervention syndicale a dû trouver son chemin dans cette intrication $\mathrm{du}$ réel et du fantasme qui structure aussi le rapport à la conduite. Ce n'est probablement pas un hasard si l'amorce de chemin s'est produite à la marge de ce milieu, dans un réseau avec un « tramway » à pneus.

\section{Du silence à l'intervention syndicale}

Si du côté des exploitants, des constructeurs et des services de l'état, il n'y a pas de réflexion sur les systèmes de veille, du côté des syndicats, il 
n'y a guère plus de réflexion sur ce dispositif, pas plus d'ailleurs que sur le renouveau du métier de conducteur de tramway. La critique du système de veille se fait à bas bruit, au sein de chaque réseau.

Ce sont d'abord des plaintes silencieuses qui se manifestent sous la forme d'orthèses pour le membre supérieur gauche (les manipulateurs traction sont situés à gauche sur tous les tramways) dont se munissent les conducteurs pour continuer à travailler. Ce sont également des plaintes qui remontent par la médecine du travail ${ }^{1}$, par la hiérarchie et les représentants syndicaux et qui aboutissent non à une remise en cause de ce système de vacma mais à le rendre supportable en redistribuant la douleur entre plusieurs dispositifs d'actionnement de la veille (à la main et au pied) ainsi qu'en jouant sur la polyvalence avec le bus.

Cette stratégie n'est possible que parce que, du côté des salariés, il y a un consentement à ce dispositif tant du point de vue de sa justification que dans les conséquences qu'il induit sur la santé. Ce double consentement est le produit, d'une part, par la formation, d'une intériorisation de la " défaillance crispée » comme réalité et, d'autre part, par l'attractivité de la conduite du tramway par rapport à celle du bus qui rend acceptable la contrainte de la vacma.

Cette attractivité du tramway se fonde pour partie sur la difficulté à rester conducteur de bus, à poursuivre la conduite de son bus au milieu de la circulation urbaine et des voyageurs (Weller, 1995; Macé, 1997). Le tramway avec son site propre et sa cabine fermée offre une alternative pour éviter que l'usure au travail ne se traduise progressivement par une inaptitude à l'emploi de conducteur (Laé, 1991; Schwartz, 1997). Obtenir la possibilité de conduire un tramway devient alors un enjeu professionnel qui dépasse les modalités concrètes d'exercice de ce travail. Cela tend à rendre supportable ce qui dans d'autres circonstances ne serait probablement pas ou moins bien toléré. Cette situation peut alors être instrumentée par les directions :

"Pour ce qui est du tramway, la RATP a mis en place une réglementation qui, de notre point de vue, met en porte à faux les médecins du travail, puisque l'entreprise considère que l'on doit être apte à la conduite d'un tramway à condition de ne pas avoir de restriction émise par un médecin,

1 Ainsi, dès 1997, alors que la ligne T1 de la RATP a été mise en service en 1992, le « Service médical du travail», (Département de la RATP, DPPPS) constate que les "troubles rhumatologiques ", terme qui à l'époque recouvrait les troubles musculo-squelettiques, sont évoqués par " $30 \%$ des agents (...) Des tendinites et des troubles musculo-squelettiques sont signalés : "Après quelques temps, je me plains de douleurs à la main gauche. Je pense que cela est dû au manipulateur" " (Avril et al., 1997). 
par exemple sur les horaires. Pour les conducteurs de bus en région parisienne, du fait de la relation avec les usagers, le tramway apparaît comme une sorte de Graal pour s'extraire de cette problématique relationnelle et des risques d'agression qui s'accroissent. C'est pourquoi ils ne déclarent pas au médecin du travail leurs problèmes de santé, de dos ou au niveau des bras ou des poignets, pour ne pas être mis en inaptitude ou ne pas avoir de restriction, parce que cela les empêcherait de conduire un tramway» (Goncalves, CHSCT RATP in Foot \& Garrigou, 2014, pp. 22-23).

Mais ce silence sur le travail des conducteurs de tramway va progressivement se briser à l'initiative d'un syndicat CGT, dans un réseau de province, celui de Clermont-Ferrand (Bernard et al., 2017). Cette initiative locale enclenche un processus de mobilisation progressif des syndicats sur le renouveau d'un métier, celui de conducteur de tramway. Cette mobilisation se manifeste au travers d'une augmentation des expertises demandées par les instances de représentation des salariés ${ }^{1}$ ou, dans le cadre d'un accord avec les directions et des études demandées aux services de santé au travail ou à des spécialistes ${ }^{2}$.

Dans cette dynamique, la Fédération des transports CGT joue un rôle central en créant les conditions d'une mutualisation des connaissances et des points de vue entre les réseaux et surtout en enclenchant, début 2012, un mouvement national contre la vacma.

La mobilisation syndicale autour de la mise en cause de la santé des conducteurs et de la sécurité de conduite par la vacma

Cette mobilisation rend plus sensible l'ensemble des acteurs aux questions de santé liées à la vacma. De nouvelles études, suite à des reconnaissances de maladies professionnelles, permettent de mettre en évidence le fait que la vacma met également en cause la sécurité de conduite (Cail et al., 2011; Franchi \& Huyghe, 2011). Il apparaît clairement que si la détection de la «mort crispée » et le contrôle de la vigilance sont des fonctions fictives, par contre, la fonction distractive ne l'est pas (Doniol-Shaw et al., 2017). L'obligation de veiller à une fréquence élevée apparaît non seulement absurde mais aussi dangereuse tant pour la santé que pour la sécurité.

1 CE du département Bus de la RATP (LATTS, 2009), CE de Reims (LATTS, 2011), CHSCT de Tours (SECAFI, 2012), CHSCT Bordeaux (Indigo Ergonomie, 2013), CHSCT Lille (7 Ergonomie/LATTS, 2014)

2 Lille (CRAM Nord-Picardie, 2007), Lyon (AGEFIPH, 2010), Strasbourg (ERCOS, 2011), Montpellier (TMS Institute, 2014), Dijon (AIST 21, 2014), Besançon (AST 25, 2016). 
Le constat est fait, dans différentes études, d'un actionnement beaucoup plus fréquent de la veille que ce qui est théoriquement nécessaire. La fréquence d'appui adoptée par la majorité des conducteurs tourne, en effet, autour de 30 à 60 actions par minute c'est-à-dire une action toutes les 1s à 2s (Cail et al., 2011; Mouchel et al., 2012; Brodbeck et al., 2013; Page, 2015). Ce calage du rythme autour du temps autorisé pour le relâchement signale un conflit entre la conduite et l'action de veiller :

«Il semble que la stratégie adoptée par les conducteurs, consistant à veiller plus fréquemment que nécessaire, soit la solution la plus économique sur le plan cognitif. Elle traduirait une charge cognitive élevée, mobilisée en partie par la temporisation de la veille telle qu'elle est prescrite. Les conducteurs adopteraient donc cette stratégie pour se concentrer sur l'environnement de conduite» (Cail et al., 2011, p. 61).

Cette analyse qui permet de comprendre que les conducteurs prennent sur eux, mettent en danger leur santé, pour assurer malgré tout la sécurité de conduite rend la lutte contre ce dispositif plus mobilisatrice. L'action s'étend à une dizaine de réseaux et passe par le vote de motions en CHSCT, le déclenchement d'alarmes sociales et des lettres adressées aux autorités organisatrices et au service technique de l'État en charge du contrôle des tramways, le STRMTG.

Si l'on s'en tient au contenu des réponses obtenues dans le cadre de ce mouvement, on pourrait penser que l'action a été vaine mais l'enjeu de cette mobilisation n'était pas là. Il s'agissait avant tout de sortir du faceà-face stérile dans lequel chaque syndicat était cantonné vis-à-vis de «sa direction. En mutualisant les connaissances, il s'agissait également d'inverser le rapport de force dans le savoir au niveau local afin d'affaiblir la suffisance souvent exprimée par les directions de réseaux sur ces sujets techniques. De plus, par le recours, en particulier aux procédures d'alarme sociale, cette mobilisation argumentative obligeait les directions à aller au-delà d'une simple fin de non-recevoir et les amenait à faire remonter ces questions au niveau national. L'interpellation du STRMTG, compte tenu de son rôle de centralisation du retour d'expérience dans l'exploitation des réseaux, avait également la même fonction.

De fait, ce mouvement avait pour enjeu de sortir des murs de chaque entreprise pour porter, dans l'espace public, à fronts renversés, une controverse opposant des syndicalistes associés à des chercheurs, soutenant une argumentation raisonnée, à des ingénieurs et responsables ferroviaires arc-boutés sur leurs fantasmes. 


\section{Avec la mort d'un voyageur, l'UTP ${ }^{1}$ propose la suppression de la vacma}

Le syndicat CGT de Montpellier, dans le cadre de la mobilisation nationale contre la vacma portée par la FNST-CGT, fait voter une motion demandant la suppression de cette vacma qui met en cause la santé des conducteurs et la sécurité de conduite en mars 2012. Le 3 septembre 2012, un freinage d'urgence (FU) déclenché par le dépassement de la limite maximum de maintien, provoque la chute d'un voyageur et sa mort.

Cet accident correspond en tout point à l'alerte donnée par les syndicalistes sur la mise en cause de la sécurité de conduite par la vacma. En effet, le déclenchement du FU est la conséquence d'une hypervigilance du conducteur sur la conduite et sur le mouvement de piétons aux limites de la voie du tramway. Concentré sur le risque d'accident, il «oublie » de relâcher la veille et n'entend pas l'alarme sonore signalant le dépassement du délai maximum de maintien autorisé.

Il a fallu la mort d'un voyageur pour que l'alerte syndicale soit prise en compte mais, probablement, sans cette alerte, tant à Montpellier qu'au niveau national, il n'y aurait pas eu cette prise en compte. Ce n'est, en effet, pas la première fois qu'il y a un accident grave voyageur résultant du déclenchement d'un freinage d'urgence. Les voyageurs victimes de chute dans la rame, consécutive à un FU, forment, en effet, la majorité des victimes du tramway et cette enquête est la première à s'intéresser à ce type d'accident ${ }^{2}$.

Le BEA TT est d'abord saisi pour procéder à une enquête sur cet accident. Puis la $\mathrm{CNESTG}^{3}$ souhaite, dès le mois d'octobre, la mise en place d'un groupe de travail sur la vacma. Kéolis, un des opérateurs internationaux les plus importants dans le transport ferroviaire urbain, filiale de la SNCF, en prend la direction pour le compte de l'UTP.

Assez rapidement, ce groupe de travail rejoint l'analyse de la CGT :

1 L'Union des Transports Publics et Ferroviaires est le syndicat patronal qui représente la profession du transport public.

2 Depuis l'accident mortel de Montpellier en 2012, un autre accident mortel similaire a eu lieu à Nice en 2015. Outre un mort, il y a eu 23 voyageurs blessés grièvement entre 2004 et 2014 suite à un freinage d'urgence. Pour les accidents légers, liés à un FU, le recensement est moins fiable mais pour la même période cela correspond à près de 3700 blessés (BEA-TT, 2016, p. $35)$.

3 La Commission Nationale d'Évaluation de la Sécurité des Transports Guidés est créée en 2003. Elle est une instance dépendant du Ministère des Transports où siègent, en particulier, des représentants du STRMTG, des autorités organisatrices, des exploitants, du syndicat patronal (UTP) et d'un constructeur. 
«La quantité d'attention actuelle que demande le dispositif VACMA peut conduire au déclenchement intempestif du freinage d'urgence (FU) et causer la chute des voyageurs. Les évolutions du dispositif actuel seront donc étudiées dans ce sens et pourront profiter à la réduction d'autres risques potentiellement induits, par exemple le risque de TMS (troubles musculo-squelettiques) 》 (cf. compte rendu de la CNESTG du 30 mai 2013).

Puis, un an plus tard, à son tour, le STRMTG partage cette analyse. En effet, suite à l'accident de Montpellier, il a procédé à une analyse plus fine des accidents voyageurs et a intégré la question de la surcharge cognitive, liée à la vacma, comme facteur d'accident :

"Il reste que la veille est une cause identifiée par les exploitants pour toutes les années depuis 2005 et qui présente pour les six dernières années une part importante des causes des victimes de freinages d'urgence. Il faut toutefois souligner que l'origine de ces absences d'activation de la veille reste imprécise. Elles pourraient être liées à l'erreur de manipulation, l'hypovigilance du conducteur ou sa surcharge cognitive » (STRMTG, Accidentologie des tramways 2012, février 2014).

Cette analyse est développée également par le groupe de l'UTP :

"L'analyse du retour d'expérience sur la VACMA soulève les questions:

1) de la mobilisation de l'attention du conducteur au détriment de sa concentration sur la conduite à vue et de sa réactivité face à l'imprévu, 2) du doute sur l'efficacité réelle du système compte tenu de l'observation d'une acquisition par les conducteurs de réflexes conditionnés qui ne permettent plus de couvrir les situations d'hypovigilance ou similaires, 3) du freinage d'urgence (FU) occasionné au détriment de la sécurité des usagers, 4) d'une accumulation de contraintes biomécaniques perturbantes; autant de d'observations qui remettent en question la pertinence du système 》 (cf. compte rendu de la CNESTG du 20 mars 2014).

Une proposition est faite de supprimer la vacma au profit d'une veille automatique, appelée, pour l'occasion, homme-mort, et l'application d'un freinage moins puissant :

"Les premiers résultats laissent entrevoir une reproduction du système d'homme mort avec application d'un freinage maximum de service (FMS) au lieu d'un freinage d'urgence (FU) » (cf. compte rendu de la CNESTG du 20 mars 2014) ${ }^{1}$.

Il est même recommandé, lors de la même réunion, à la suite des débats qui ont eu lieu après la présentation des travaux du groupe de travail, "que le groupe de travail consulte à un moment donné les organisations syndicales actuellement très concentrées sur le sujet de la veille ».

1 Lors d'un entretien téléphonique avec le responsable de la sécurité de Keolis qui s'exprimait lors de cette réunion au nom du groupe de travail de l'UTP sur la fonction de veille, celui-ci m'a confirmé que le terme " système d'homme-mort » servait à désigner un dispositif de veille que l'on peut maintenir en permanence, donc sans temporisation au maintien. Il avait utilisé cette dénomination pour le distinguer de la vacma. Il préconisait donc la suppression de la vacma et son remplacement par une veille automatique/homme-mort. 
Pourtant, rien ne se débloque officiellement. Il n'y a pas plus d'ouverture de discussion avec les syndicats que de recommandations faites pour remplacer la vacma par une veille automatique, une veille à appui maintenu.

Toutefois, s'il n'y a pas de décision officielle au niveau national, au niveau de deux réseaux, Montpellier et Clermont-Ferrand, des discussions sont engagées avec les directions locales. Ces deux réseaux symbolisent le bien-fondé de la critique de la vacma portée par la CGT. Pour le premier, l'accident mortel d'un voyageur confirme l'insécurité de conduite produite par ce système de "sécurité " autant que, pour le second, la reconnaissance de huit maladies professionnelles manifeste le caractère pathogène de la vacma. Ces discussions débouchent sur la mise en place d'une veille automatique, i.e. sans contrainte de relâchement périodique en roulant, en 2017, sur deux lignes pour Montpellier et, en 2016, pour Clermont-Ferrand.

Ces avancées peuvent sembler locales mais, de fait, elles correspondent également à la production d'une décision nationale puisque, à chaque fois, le STRMTG est amené à donner son accord pour la mise en place de ces nouveaux dispositifs de veille, à dire que ce système respecte le principe de "non régression de la sécurité ». Chaque victoire locale constitue de ce fait une jurisprudence technique sur laquelle d'autres réseaux, que ce soit au niveau syndical ou des directions, peuvent s'appuyer pour transformer leur situation.

Ces histoires particulières manifestent l'affaiblissement du consensus technique autour de la vacma dans le milieu du tramway mais sans, toutefois, pouvoir déboucher de manière globale.

\section{Rester entre soi pour préserver sa « folie »}

De manière symptomatique, à partir du moment où les responsables du groupe de travail, des cadres techniques, émettent en 2014, le souhait d'une discussion avec les syndicalistes, ce sont les responsables des relations sociales qui reprennent la main. Dès lors, il ne sera plus question ni de consultation ni même de discussion. Du point de vue de l'UTP, ces questions «techniques» sur la veille n'affectent pas «le contenu du métier $»{ }^{1}$.

1 Lettre du 10/01/2014 du Délégué Général de l'UTP au secrétaire fédéral de la CGT Transports en réponse au courrier du 18/12/2013. 
Cette exclusion des syndicats des discussions qui ont cours dans le milieu du tramway est renforcée par le fait que les seuls interlocuteurs légitimes du groupe de travail de l'UTP apparaissent être "les constructeurs de matériel roulant $»$.

Cette volonté d'exclure les représentants des conducteurs des discussions sur les conditions techniques du travail de conduite est d'autant plus paradoxale que, depuis le début, la production de connaissance sur le système de veille a été faite à l'initiative de syndicalistes et, souvent, avec le concours des Carsat. Ainsi en a-t-il été pour la remise en cause de l'hypothèse de la «mort crispée » du «contrôle de vigilance» ou pour la mise en évidence d'une fonction distractive des actions sur la veille par rapport à la conduite.

L'irrationalité du point de vue du milieu sur la veille a atteint un paroxysme avec une prescription pour la conception d'une pédale de veille automatique qui voulait que "le conducteur, une fois le pied posé sur la veille, ne produit pas d'effort pour maintenir la pédale enfoncée dans cette position » (STRMTG, 2012, p. 13). Cette prescription, aboutissement d'un travail collectif du milieu tramway ${ }^{1}$, n'a étonné personne alors qu'il est quand même paradoxal de trouver fonctionnel que le poids «mort» d'une jambe puisse indiquer que le conducteur est vivant. Ce paradoxe est d'autant plus troublant que la crainte qui hante le milieu est celle d'une « mort crispée », d'une mort qui fait un effort ! Là aussi, l'alerte sur cette absurdité dangereuse a été la conséquence d'une initiative syndicale ${ }^{2}$.

Fait remarquable, cette alerte a été entendue et prise en compte assez rapidement puisque le guide d'ergonomie des postes de conduite a été

\footnotetext{
$1 \mathrm{La}$ règle de base du groupe de travail animé par le STRMTG est que «Chaque participant exploitant doit se considérer comme représentant du groupement auquel il appartient, et doit ainsi jouer le rôle de porte-parole et de courroie de transmission. Certains réseaux "indépendants" n'étant pas représentés, il appartient à l'UTP de jouer ce rôle ». (Menétrieux, STRMTG, GT REX Tramways. Journée UTP du 15/06/2010).

2 L'alerte a été donnée dans le cadre d'une expertise CHSCT (Elwert et al., 2013). Elle s'est appuyée sur le rapport de la commission d'enquête sur l'accident de Waterfall, en Australie, qui a fait, en 2003, 7 morts et 42 blessés. Cet accident a pu avoir lieu car, malgré la mort du conducteur, "il a pu maintenir une force suffisante sur la pédale, due au "poids mort" de sa jambe, pour la garder en position conduite " (MCInerney, 2005, p. 14). Le processus qui a abouti à ce résultat est analogue à celui qui a permis au STRMTG de produire une prescription absurde : "L'attention a été portée, pour la reconception de la pédale, sur le confort et la réduction des TMS du membre inférieur, même s'il avait été dit que la sécurité avait le pas sur les autres facteurs » (MacIntosh \& Edkins, 2007). Personne n'a prêté attention au fait que les conducteurs «ont également signalé que la pédale est rarement désactivée quand ils sont somnolents ou inattentifs » (idem).
} 
réécrit de fond en comble à peine trois ans après sa première publication ${ }^{1}$ (STRMTG, 2015). Pourtant, bien que dans cette refonte du guide, les expertises demandées par les IRP (instance de représentation des salariés) aient contribué dans une large mesure à ce processus, la composition du milieu en charge de cette réécriture reste identique, toujours aussi exclusive vis-à-vis des représentants des conducteurs.

Il ne s'agit pas seulement de ne pas consulter les syndicats pour produire des prescriptions organisant les conditions de travail des conducteurs mais il faut également refuser tout débat public avec des syndicalistes, même dans le cadre d'un colloque scientifique. Cela se traduit, en particulier, par un boycott du colloque scientifique organisé en 2014, à l'initiative de la FNST-CGT, sur les thèmes de la veille et des conditions de travail des conducteurs de tramway (Foot \& Garrigou, 2014).

Si l'on en croit le secrétaire général de Transdev, ce refus correspond bien à une décision politique de l'UTP :

" CGT: L'UTP était invité à ce colloque mais je n'ai vu aucun représentant.

Secrétaire général Transdev: Je suis étonné car lorsque l'invitation de la CGT nous est parvenue, j'ai proposé que l'UTP accepte l'invitation. Et donc l'UTP devait participer à ce colloque » (Compte rendu de la réunion du 27/06/14 du Comité de Branche France de Transdev).

Ce refus de tout dialogue fait également l'objet de discussions dans des instances où les syndicats sont absents. Ce qui signale probablement que cette position ne fait pas l'unanimité au sein du milieu du tramway. Ainsi, lors de la réunion du 17 juillet 2014 du groupe de travail pour la mise à jour du guide d'ergonomie, le STRMTG invoquera un argument spécieux pour justifier son absence, le fait que «son champ de compétence ne couvre pas la santé des travailleurs ». Pourtant, cela ne semble pas l'empêcher de prendre en charge la publication d'un guide d'ergonomie ! À cette même réunion, en contrepoint de cette position, le représentant de Keolis rappelle «qu'à la CNESTG de mars 2014, il a été annoncé qu'une concertation avec les conducteurs était prévue dans le cadre du GT Veille» (Compte-rendu du 23/07/14, document STRMTG).

Ce refus du dialogue manifeste une stratégie dominante du milieu du tramway qui tient à tenir à l'écart les conducteurs et leurs représentants comme si l'intervention de ces derniers pouvait mettre en danger la capacité de ce groupe à rester un groupe structurant du milieu. La clôture du milieu sur lui-même s'apparenterait alors à une stratégie défensive

1 La prescription sur les dispositifs de veille n'était pas le seul lieu où des prescriptions étaient inadaptées voire impraticables. Les expertises demandées par les IRP ont été des moments de mise à l'épreuve de la pertinence du guide. 
mise en place pour préserver sa manière de penser, pour protéger son idéologie ferroviaire spécifique contre toute intervention du " dehors». Cette clôture s'apparente à une coupure de ce groupe du réel de l'activité des conducteurs. C'est "la situation d'un groupe ou d'un sous-groupe social dans lequel l'impératif de solidarité entre ses membres (relation ego-autrui) est placé au-dessus de la prise en considération du réel qui est la raison d'être du groupe » (Sigaut, 1990).

\section{Tourner autour du mot pour dire sans vraiment dire}

Cette stratégie de coupure mise en œuvre par ce groupe, i.e. le groupe de travail «REX Exploitation Tramway» et les autres groupes de travail associés du type GT «Fonction Veille», qui formalise, par sa production, (guides techniques, guides d'application, bases de données, études et rapports), le rapport technique du milieu au tramway, se manifeste dans l'invention d'un lexique spécifique. Ce jargon professionnel singularise, dans cet espace de la technique et du travail, le langage commun. Il subvertit le sens de mots et trouble alors la communication dans le milieu tout entier. Ce groupe social "produit des systèmes de normes socio-linguistiques qui règlent ce qui peut être dit, à qui, comment et dans quelles situations » (Boutet, 1994, p. 62). Il tend à imposer, en mettant en avant le nouveau sens des mots, un changement du sens de la technique, un changement du sens de l'action elle-même'

Cette stratégie se révèle en particulier autour de la vacma.

Le terme de vacma, invention linguistique de la SNCF des années 1960, s'est imposé dans le monde ferroviaire y compris le ferroviaire urbain. Ainsi, lors de la "Journée d'échanges tramway» organisé par le STRMTG en 2014, c'est ce terme de vacma qui est utilisé par les participants. Ce terme est également mobilisé dans les rapports du BEA TT ou les réunions de la CNESTG. On le retrouve aussi dans les rapports d'accidentologie du STRMTG, depuis le rapport de février 2014, ou encore dans la présentation du " groupe Fonction veille » par l'UTP lors de la Journée d'échanges tramway de mai 2016. Bref, dans les pratiques langagières ordinaires de ce milieu du tramway, le terme de vacma est d'usage courant

Pourtant, que ce soit dans la première version de 2012 du guide d'ergonomie du STRMTG ou dans sa troisième version de 2015 ou

1 La technique avant d'être un objet est avant tout action. Il peut y avoir des techniques sans objet mais pas sans action (Sigaut, 1990; Mauss, 1999). 
encore dans le guide «Fonction veille » de 2017, on ne trouve pas trace du terme de vacma. Le terme de vacma est remplacé par les expressions de « veille », " veille à appuis répétés » ou « commande vigilance ».

Avec cette opération de substitution, on passe d'un acronyme, celui de « vacma », qui résume le programme d'action de ce dispositif, une veille automatique qui contrôle le mode de maintien de l'appui, dont la fonction est d'empêcher la fraude, à une définition qui décrit simplement le fonctionnement d'un dispositif sans souci de sa fonction.

Dans ce glissement sémantique, on constate une inversion de la description $\mathrm{du}$ fonctionnement de ce dispositif. Avec la vacma, on contrôle le mode d'appui par une obligation de relâchement périodique de la commande. Si l'on suivait la nouvelle manière de décrire cet objet, si on le nommait à partir de son fonctionnement, on parlerait de la vacma comme d'une «veille à relâchement répété». Dans l'oubli de la fonction, la description du fonctionnement s'inverse, l'appui se substitue au relâchement comme si, dorénavant, c'était le mode d'obtention du relâchement qu'il fallait contrôler, comme si la hantise de la «mort crispée » avait définitivement pris le pas sur celle de la fraude ${ }^{1}$.

En 2015, avec la nouvelle version du Guide, on revient à la définition fonctionnelle originelle de 1942, celle d' " un dispositif permettant de stopper la rame en cas de perte de connaissance du conducteur " (Guide 2015) mais l'expression de "veille à appui répété» est encore reprise. Ce nouveau guide corrige des éléments qui ont fait l'objet d'alertes. La définition de la veille comme "commande vigilance» disparaît et le terme de vigilance n'est plus employé. Elle corrige également la prescription précédente, qui demandait de pouvoir «maintenir sans effort $»$ la veille. Dorénavant, des veilles qui pourraient être maintenues avec une «main tombant sur un bouton" ou par "le poids propre d'une jambe », «ne sont pas acceptables ».

Mais, elle poursuit la dérive du sens associé à la veille, en substituant le terme d'acquittement (veille acquittée, non acquittement de la veille,

\footnotetext{
1 Cette inversion du sens est explicitée ainsi par une des responsables du STRMTG au cours de la journée du 13 mai 2016 : «la temporisation de maintien était à l'origine pour permettre la détection d'une électrocution du conducteur via le manipulateur, risque considéré comme mineur aujourd'hui. Il a par ailleurs été observé qu'un malaise conduisait plutôt à un relâchement $d u$ corps ». On notera qu'il n'est nulle part fait mention de la détection de la fraude par le relâchement. Le "mythe ferroviaire» de l'électrocution est, à cette occasion, réactivé autant qu'actualisé sous la forme d'un "risque mineur » tandis que le "relâchement » est "plutôt» une tendance du corps en cas de défaillance qu'un symptôme identifié. Les fonctions du maintien et du relâchement vis-à-vis du dispositif de veille restent donc incertains pour le STRMTG.
} 
position acquittée, acquitter la veille, Guide 2015) à celui d'activée (vigilance activée, activation de la vigilance, Guide 2012). Or, cette substitution est, dans le monde ferroviaire, étonnante car il est d'usage de considérer qu'on acquitte un signal, une alarme mais ce terme n'est jamais utilisé pour une action nominale sur une commande, ce qui, en théorie, est le cas pour la veille. Pour le STRMTG, ce sens est d'ailleurs celui retenu pour parler d' " acquittement alarme Matériel Roulant» ou d' " acquittement poignée d'alarme » dans le Guide 2012 (p.15) et 2015 (p.16 et 22).

Avec le terme d'acquittement, la conception de la veille loin de se clarifier poursuit sa capacité à produire des malentendus sur le sens de l'action de veiller.

L' «aliénation culturelle » (Sigaut, 1990) de ce groupe prescripteur du milieu du tramway se boucle alors sur le langage et rend manifeste sa coupure d'avec les conducteurs de tramway, avec le réel de la conduite. Le maintien de l'existence de ce groupe passe par «sa clôture - la frontière étant la langue » (Nathan, 1994, p. 182).

Tout cet effort sémantique - passer de «vacma» à «commande vigilance» puis à «appui répété», passer de «activation» à " acquittement» puis à "réarmement» - pour transformer la fonction de la veille sans jamais, paradoxalement, le dire, aboutit à une perte d'intelligibilité du sens de l'action de ce groupe prescripteur du rapport technique au tramway. Ce travail aboutit à un langage paradoxal où les propositions se contredisent mais pourtant coexistent. Les mots ne sont plus susceptibles de soutenir la réussite de l'action.

\section{Cela ne peut-il donc pas se conclure?}

Le dénouement semble proche où l'on pourra remiser la vacma des tramways au musée des bizarreries technologiques. En effet, à la suite de l'accident de Montpellier, les différents acteurs du milieu tramway semblent converger pour mettre en cause cette vacma. Ce n'est pas la santé des conducteurs qui forme l'argument principal de cette mise en cause mais la sécurité des voyageurs.

Entre 2014 et 2016, la «surcharge cognitive » change de statut pour l'UTP et le STRMTG. D'hypothèse, cette surcharge est devenue un problème réel. L'UTP propose alors une «clarification des objectifs de la fonction de veille : réduire la surcharge cognitive du conducteur de tramway afin de se concentrer sur la "conduite à vue" " (Présentation 
$\mathrm{du}$ 10/05/16, UTP). La transformation du dispositif de veille doit permettre que le conducteur ait «une attention mieux focalisée sur la conduite proprement dite ».

Enfin, le STRMTG publie en janvier 2017, un guide « Fonction de veille des tramways. Exigences de sécurité » (STRMTG, 2017) dans lequel le bien fondé des arguments mobilisés par la FNST-CGT est reconnu tant sur le conflit cognitif entre la veille et la conduite que sur le symptôme de la défaillance pris en compte par ce type de dispositif de veille :

La VACMA produit de l'insécurité pour la conduite

"L'analyse du retour d'expérience a relevé de nombreux FU veille déclenchés alors que le conducteur est présent et conscient. Ces déclenchements à tort sont dus [...] à de la saturation cognitive (hypervigilance). En effet, l'attention du conducteur est fortement sollicitée en environnement urbain ce qui peut entraîner une surcharge cognitive conduisant à l'oubli d'activation du dispositif de veille. Ces freinages d'urgence conduisent à un certain nombre de chutes de voyageurs dans la rame alors qu'aucun danger imminent n'est avéré » (p.4).

Le relâchement de la veille est le seul signe pris en compte pour détecter une défaillance

«L'hypothèse est faite que le malaise du conducteur conduit à un relâchement de ses membres » (p.7).

La défaillance « crispée » n'est pas un risque réel

«les malaises conduisant à une crispation des membres ne sont plus pris en compte » (p.7)

En conséquence de quoi, la temporisation au maintien pendant la conduite n'a plus, explicitement, de justification fonctionnelle. On peut donc supprimer la vacma et lui substituer, comme cela a déjà été fait à Clermont-Ferrand et Montpellier, un système de veille sans temporisation au maintien que le conducteur peut maintenir continûment pendant la conduite. Il peut également le relâcher quand il le veut dans les mêmes conditions que précédemment c'est-à-dire durant 3 secondes sans alarme sonore et 2 secondes avec alarme sonore. La seule contrainte au relâchement de la veille se situe à l'arrêt, en station, "l'autorisation de départ d'une station devra être conditionnée à un cycle d'appuirelâchement $\gg$ (p.10).

Tout semble donc se conclure avec la publication de ce guide qui reconnaît que la vacma, dispositif de sécurité, est une source d'accident permanente en milieu urbain. À la suite de ce qui s'est déjà fait dans deux réseaux, il paraît logique que cette substitution se généralise 
puisque non seulement cela ne crée pas de régression de la sécurité mais, en éliminant pour une part importante, le conflit cognitif entre la veille et la conduite, cela améliore la sécurité de conduite.

L'action syndicale serait donc parvenue à rétablir ce groupe prescripteur, au moins pour ce qui concerne la veille, dans un rapport apaisé au réel.

Pourtant, ce n'est pas le cas. Il n'est fait aucune obligation ni de transformer les systèmes existants, ni même les systèmes futurs alors que la dangerosité de la vacma est explicitement reconnue.

Pis encore, le milieu du tramway est prêt à dégrader la sécurité du dispositif de veille pour «sauver » la vacma.

Ainsi, il accepte que des actionneurs de veille soient inefficaces et, plutôt que de les rendre fonctionnels, il triple le temps de détection d'une défaillance possible qui passe de 5 à 15 secondes.

Ainsi, pour compenser la surcharge cognitive provoquée par la vacma, il est désormais possible d'allonger la temporisation au relâchement afin que, éventuellement, le conducteur puisse rattraper un «oubli» d'actionnement de la veille provoqué par la vacma.

Toutes ces mesures qui complexifient un dispositif dont il est reconnu qu'il n'a non seulement aucun intérêt fonctionnel mais que, de plus, il contribue à dégrader la sécurité de conduite, font penser au Monsieur K de Brecht qui se donnait de la peine pour préparer sa prochaine erreur. Là, le milieu de la sécurité du tramway semble perdre pied et ne plus pouvoir comprendre le sens de son action.

D'un côté, il est affirmé que la vacma dégrade la sécurité et, par conséquent, on peut la supprimer, de l'autre, on dégrade la sécurité pour pouvoir conserver malgré tout la vacma. Le milieu de la sécurité du tramway semble traversé par un clivage qui lui permet de faire coexister ces deux modes d'existence contradictoires de la vacma.

Tout se passe comme si le milieu se clivait pour pouvoir préserver sa possibilité de conserver son aliénation au réel, de préserver sa folie.

Robin Foot

Sociologue, ingénieur de recherche Cnrs Laboratoire Techniques, Territoires et Sociétés 


\section{Bibliographie}

Akrich, M., 1987, Comment décrire les objets techniques? Techniques et Culture(9), 49-64.

Amalberti, R., 1996, La conduite de systèmes à risques. Paris: PUF.

Arendt, H., 1991, Eichman à Jérusalem : rapport sur la banalité du mal Paris: Gallimard.

Avril, G., Lerner, M., Leton, M., Matteï, N., \& Delattre, A., 1997, Étude de poste : Machiniste-receveur sur le tramway - Ligne T1 (pp. 90). Paris: Service Médical du Travail - DPPPS - RATP.

BEA-TT, 2005, Rapport d'enquête technique sur l'accident de tramway survenu à Rouen le 30 août 2004 (pp. 82). Paris: Bureau d'Enquêtes sur les Accidents de Transport Terrestre.

BEA-TT, 2011, Rapport d'enquête technique sur le tamponnement de deux rames de tramway survenu le 12 mai 2010 à Montpellier (34) (pp. 70). Paris: Bureau d'Enquêtes sur les Accidents de Transport Terrestre.

BEA-TT, 2016, Rapport d'enquête technique sur la chute mortelle d'un voyageur dans un tramway lors d'un freinage d'urgence le 3 septembre 2012 à Montpellier (34) (pp. 48). Paris: Bureau d'Enquêtes sur les Accidents de Transport Terrestre.

Bernard, B., Desmarie, C., \& Hillaire, J.-M., 2017, 13 ans de lutte contre les TMS. Travailler(38).

Billiard, I., 2001, Santé et mentale et travail. L'émergence de la psychopathologie du travail. Paris: La Dispute.

Boutet, J., 1994, Construire le sens. Berne: Peter Lang.

Brodbeck, C., Carballeda, G., Cheikh, S., Chotin, P., Clerici, C., Garrigou, A., et al., 2013, Évaluation des conditions de travail des traminots favorisant l'exposition à des risques professionnels et en particulier le risque de TMS, Expertise CHSCT de Keolis Bordeaux (pp. 282). Pessac: Indigo Ergonomie.

Cail, F., Morel, O., \& Aublet-Cuvelier, A., 2011, Quantification des contraintes biomécaniques de 4 conducteurs de tramway de T2C (pp. 66): INRS.

CGT, 1966, Conférence sur les conditions de travail des agents de conduite. Paris: Fédération nationale des cheminots CGT.

Couëdel, G., \& Nouailhetas, C., 2017, Retour sur la Vacma à la SNCF et l'action de la CGT : la parole de deux syndicalistes. Travailler(38).

Dejours, C., 1993, Travail, usure mentale. Suivie d'un addendum théorique : De la psychopathologie à la psychodynamique du travail. Paris: Bayard.

Doniol-Shaw, G., \& Foot, R. (Eds.), 2004, Travail de conduite et sécurité des tramways : enjeux pour la conception du poste de conduite. Marne-laVallée: LATTS/T2C.

Doniol-Shaw, G., Foot, R., \& Franchi, P., 2017, Une mise en veille de la santé et de la sécurité ou les paradoxes du dispositif d'Homme mort sur les tramways. Travailler(38).

Doniol-Shaw, G., Foot, R., \& Zembri, P., 2010, Conduite du Translohr : Réflexions sur un processus de concertation et sur la conduite d'un tramway \& Les lignes T5 et T6 : Analyse des tracés, de la charge, des 
sites et de l'environnement réglementaire Rapport d'expertise nouvelles technologies pour le CDEP Bus de la RATP (pp. 185). Marne-laVallée: LATTS.

Elwert, L., Florence, E., \& Foot, R., 2013, Rapport d'expertise pour le CHSCT de $\mathrm{T} 2 \mathrm{C}$ sur le projet de passage d'une vacma manuelle à une veille automatique au pied sur le Translohr (pp. 148). Nancy: 7 Ergonomie.

Elwert, L., \& Foot, R., (2015, 23-25 septembre 2015). Du conflit à la coopération, objets intermédiaires et médiateurs au coeur de la conception d'un poste de conduite de tramway. Communication présentée au 50ème Congrès de la SELF, Paris.

Favret-Saada, J., 1985, Les mots, la mort, les sorts. Paris: Gallimard.

Foot, R., 1991, Le voyageur, l'électricité et le conducteur Savoir-faire et pouvoir transmettre, Ethnologie de la France, cahier $n^{\circ} 6$ (pp. 133149). Paris: Editions de la MSH.

Foot, R., \& Garrigou, A. (Eds.), 2014, Homme mort et conditions de travail des conducteurs de tramway. Actes du colloque organisé par le LATTS, le LSTE, la FNST CGT et Indigo Ergonomie le 23 juin 2014. Marne-laVallée: Université Paris Est.

Franchi, P., \& Huyghe, D., 2011, T2C : Prévention des TMS chez les conducteurs de tramway (pp. 102): Idénéa Ergonomie.

Garreau, M., \& Laplaiche, M., 1942, Rapport de M.M. Garreau et Laplaiche sur la question de l'homme mort appliqué aux locomotives électriques (pp. 28).

Henry, E., 2007, Amiante un scandal improbable. Sociologie d'un problème public. Rennes: PUR.

Laé, J.-F., 1991, L'inaptitude à la RATP, de la protection à la sanction. Sociétés contemporaines, 8(1), 107-125.

Latour, B., 1984, Les Microbes : guerre et paix, suivi de Irréductions. Paris: A.-M. Métailié.

Latour, B., 1994, Une sociologie sans objet ? Remarques sur l'interobjectivité. Sociologie du Travail, 94( $\mathrm{n}^{\circ}$ spécial sur la cognition située), 587-606.

Latour, B., 2015, Face à Gaïa. Huit conférences sur le nouveau régime climatique. Paris: Les empêcheurs de penser en rond/La Découverte.

Law, J., 1992, Notes on the theory of the actor-network: Ordering, strategy, and heterogeneity. [journal article]. Systems practice, 5(4), 379-393. doi: $10.1007 / \mathrm{bf0} 1059830$

Le Guillant, L., Pariente, M., Kipman, \& Moscovitz, J.-J., 2006, Réflexions sur une condition de travail particulièrement pénible : la Vacma In L. Guillant (Ed.), Le drame humain du travail (pp. 149-167). Ramonville Saint-Agne: Érès.

Llory, M., 1996, Accidents industriels : le coût du silence. Opérateurs privés de parole et cadres introuvables. Paris: L'Harmattan.

Macé, É., 1997, Service public et banlieues populaires : une coproduction de l'insécurité Le cas du réseau busde la RATP. Sociologie du Travail, 39(4), 473-498.

MacIntosh, A., S, \& Edkins, G., 2007, The Waterfall train accident implications and lessons learnt. In J. Wilson, R, B. Norris, T. Clarke \& A. Mills (Eds.), People and Rail Systems: Human Factors at the Heart 
of the Railway (2nd European Rail Human Factors Rail Conference, 2005) (pp. 148-153). Hampshire: Ashgate.

Magritte, R., 1994, Les mots et les images. Bruxelles: Labor.

Mauss, M., 1999, Les techniques du corps Sociologie et anthropologie (pp. 363-386). Paris: Quadrige/PUF.

MCInerney, P., Aloysius, 2005, Special Commission of Inquiry into the Waterfall Rail Accident. Final report (Vol. Volume I, pp. 483).

Molinier, P., 2008, Les enjeux psychiques au travail Paris: Payot.

Mollard, R., Coblentz, A., \& Cabon, P., 1991, Détection de l'hypovigilance chez les conducteurs de train Le maintien de la vigilance dans les transports. Journée d'étude de l'INRETS (pp. 65-71). Caen: Paradigme.

Moscovitz, J.-J., 1970, À propos d'une rencontre avec les roulants de la S.N.C.F. Les Temps Modernes, 289, 507-519.

Moscovitz, J.-J., 1972, Approche psychiatrique des conditions de travail. Entretiens psychiatriques(15).

Mouchel, M., Anceaux, F., Fourez, E., \& Miglianico, D., (2012). Lien entre stimulation environnementale et vigilance des conducteurs de tramways : une analyse ergonomique. Communication présentée au 18ème Colloque National de Maîtrise des Risques et Sûreté de Fonctionnement, Tours.

Nathan, T., 1994, L'influence qui guérit. Paris: Odile Jacob.

Page, É., 2015, Refonte ergonomique du poste de conduite des tramways de Clermont-Ferrand. (Mémoire de stage scientifique), École des Ponts ParisTech, Marne-la-Vallée.

Paillard, J., 1976, Tonus, postures et mouvements. In C. Kayser (Ed.), Physiologie. Livre deuxième: système nerveux. Muscles (3ème ed., pp. 521-728). Paris: Flammarion.

Peter, J. H., Fuchs, E., Langanke, P., Meinzer, K., \& Pfaff, U., 1983, The SIFA train function safety circuit. I Vigilance and Operational Practice in Psycho-physiological Analysis. International Archives of Occupational and Environmental Health, 52(4), 329-339.

Proctor, R., N. , 2014, Golden holocaust. La conspiration des industriels du tabac. Paris: Équateurs.

Ribeill, G., 1997, Les conducteurs électriciens : tatonnements et lenteurs autour de la reconnaissance d'un nouveau métier. Revue d'histoire des chemins de fer(Hors série n 5 ), 385-398.

Ribeill, G., 2017, Charles Walbrou, « Je préférerais ne pas », le réfractaire de 1'Homme Mort. Travailler(38).

Schwartz, Y., 1997, Sur la question corporative dans le mouvement social de décembre 1995. Sociologie du Travail, 39(4), 449-471.

Sigaut, F., 1990, Folie, Réel et technologie. Techniques et culture, 15, 167-179.

STRMTG, 2012, Ergonomie des Postes de Conduite des Tramways - Cahier des Charges - Guide technique (pp. 22). Saint Martin d'Hères: Service technique des remontées mécaniques et des transports guidés - version 1.

STRMTG, 2015, Sécurité des Postes de Conduite des Tramways - Version 3 Guide technique (pp. 27). Saint Martin d'Hères: Service technique des remontées mécaniques et des transports guidés. 
STRMTG, 2017, Fonction de veille des tramways. Exigences de sécurité Guide technique (pp. 12). Saint Martin d'Hères: Service technique des remontées mécaniques et des transports guidés.

Terssac de, G., \& Mignard, J., 2011, Les paradoxes de la sécurité. Le cas AZF.Thomas, L.-V., 1980, Anthropologie de la mort. Paris: Payot.

Weller, J.-M., 1995, Le machiniste receveur et les voyageurs, expertises et apprentissages d'une relation (Vol. 103). Paris: RATP-Département du Développement/Mission Prospective.

Wisner, A., 1985, Quand voyagent les usines. Paris: Syros. 